\title{
Plasticidade cortical e técnicas de fisioterapia neurológica na ótica da neuroimagem
}

\author{
Cortical plasticity and neurological physical therapy techniques in neuroimage optic
}

\section{Gabriela Barato', Tatiana Fernandes ${ }^{1}$, Mariana Pacheco², Victor Hugo Bastos ${ }^{3}$, Sergio Machado ${ }^{4}$, Mariana Pimentel de Mello5, Júlio Guilherme Silva ${ }^{6}$, Marco Orsini ${ }^{7}$}

\begin{abstract}
RESUMO
O acidente vascular encefálico (AVE) gera um impacto nas estruturas e funções do corpo, prejudicando a independência funcional dos indivíduos afetados. Novas abordagens conceituais têm permitido uma nova visão do sistema nervoso como um órgão dinâmico, constituindo uma unidade funcional com o corpo, o ambiente e características plásticas, através do exercício funcional em diferentes contextos. Portanto, o objetivo deste estudo foi verificar a dinâmica da reorganização do sistema nervoso central após a aplicação de técnicas de fisioterapia neurológica, particularmente, Terapia de Restrição e Indução do Movimento, Observação de Ação e Prática Mental destinadas à recuperação funcional de sujeitos com membros superiores hemiparéticos pós-AVE por meio de estudos que utilizaram imagens de ressonância magnética funcional. Conforme observado nos estudos selecionados, os resultados referentes à ativação cerebral mostraram-se inconsistentes. Portanto, conclui-se que o cérebro encontra diferentes vias para sua reorganização. Este fato deve-se possivelmente as diferentes áreas lesionadas e também devido às diferentes características de cada técnica de reabilitação utilizada nos estudos.
\end{abstract}

Unitermos: Acidente Vascular Encefálico. Membro Superior. Plasticidade Neuronal. Ressonância Magnética.

Citação: Barato G, Fernandes T, Pacheco M, Bastos VH, Machado S, Mello MP, Silva JH, Orsini M. Plasticidade cortical e técnicas de fisioterapia neurológica na ótica da neuroimagem.

Trabalho realizado na Pós-graduação em Fisioterapia Neurológica da Universidade Gama Filho, Rio de Janeiro, RJ, Brasil.

1. Fisioterapeuta, Especialista em Fisioterapia Neurofuncional, pela Universidade Gama Filho - UGF, Rio Grande do Sul.

2. Fisioterapeuta, Colaboradora do Instituto Brasileiro de Biociências Neurais - IBBN, Rio de Janeiro, RJ.

3. Fisioterapeuta, Doutorando em Saúde Mental, Instituto de Psiquiatria (IPUB) da Universidade Federal do Rio de Janeiro - UFRJ, Professor Pesquisador do Centro Universitário Metodista do Rio, Professor-pesquisador do Instituto Brasileiro de Biociências Neurais (IBBN-RJ), Professor da Pós-Graduação em Fisioterapia Neurofuncional (UGF-RS), Rio de Janeiro, RJ.

4. Educador Físico, Doutorando em Saúde Mental (IPUB, UFRJ), Professor-pesquisador do Instituto Brasileiro de Biociências Neurais (IBBN-RJ), Rio de Janeiro, RJ.

5. Fisioterapeuta, Aluna do Programa de Iniciação Científica do Serviço de Neurologia - UFF, Niterói, RJ.

6. Fisioterapeuta, Doutorando em Saúde Mental (IPUB/UFRJ), Coordenador da Pós-Graduação em Fisioterapia Neurofuncional (UGF-RS), Professor-pesquisador do Instituto Brasileiro de Biociências Neurais (IBBN-RJ), Rio de Janeiro, RJ.

7. Graduando em Medicina, Doutorando em Neurologia (Universidade Federal Fluminense - UFF), Professor Titular do Programa de Iniciação Científica da Escola Superior de Ensino Helena Antipoff (ESEHA), Niterói, RJ.

\section{SUMMARY}

The stroke generates an impact in the body's structure and function, affecting the individual's functional independence. New conceptual approaches has allowed a new view of the nervous system as a dynamic organ, constituting a functional unit with the body; the environment and plastic characteristics, producing structural modifications derive from functional exercises in different contexts. Therefore, the objective of this study was to check the dynamic of the central nervous system reorganization after the application of neurological physical therapy techniques, particularly, Constraint Induced Movement Therapy, Action Observation and Mental Practice, bounded for functional recovery of subjects with hemiparetic upper limb post-stroke through studies that used images from functional magnetic resonance imaging. According to selected studies, the results related to brain activation appeared to be inconsistent. Therefore, it appears that the brain finds different routes for its reorganization. This fact should be possibly the different injured areas and also due to different characteristics of each rehabilitation techniques used in the studies.

Keywords: Stroke. Upper Extremity. Neuronal Plasticity. Magnetic Resonance.

Citation: Barato G, Fernandes T, Pacheco M, Bastos VH, Machado S, Mello MP, Silva JH, Orsini M. Cortical plasticity and neurological physical therapy techniques in neuroimage optic.

Endereço para correspondência: Sergio EC Machado

Rua Prof. Sabóia Ribeiro 69/104 20950-050 Rio de Janeiro, RJ

E-mail: secm80@yahoo.com.br 


\section{INTRODUÇÃO}

O acidente vascular encefálico (AVE) é uma das maiores causas de limitação funcional no mundo'. Devido ao envelhecimento da população mundial, o número de pessoas acometidas pelo AVE vem aumentando substancialmente ao longo dos anos ${ }^{2}$. Estudos apontam que o AVE é a terceira causa mais comum de morte em países desenvolvidos e uma das maiores causas de incapacidade, causando grande impacto na saúde mundial ${ }^{3}$. Os sintomas mais comuns são paresia ou paralisia repentina da face, membro superior e/ou inferior de um hemicorpo. Outros sintomas incluem dor de cefaléia sem causa definida, confusão mental, perda de consciência, dificuldades na fala ou na compreensão, alterações visuais, dificuldade de transferência de peso, marcha e coordenação. Os efeitos dependem da área lesada do Sistema Nervoso Central (SNC) e da forma como este é afeta$\mathrm{do}^{2}$.

A neuroplasticidade pode ser definida como a capacidade de adaptação da estrutura e função do sistema nervoso em decorrência dos padrões de experiência. Há uma década parecia inimaginável para os neurocientistas a plasticidade no córtex cerebral de adultos, entretanto, com o avanço de pesquisas e métodos de imagem utilizando técnicas não-invasivas, tal panorama vem se modificando, pois estas demonstram a tendência de sinapses e circuitos neuronais se modificar em virtude às atividades no SNC maduro afetado ${ }^{4,5}$. Mudanças plásticas ocorrem após um insulto neurológico, incluindo o AVE, em resposta à excitabilidade diminuída e ao não uso do membro afetado, reduzindo o tamanho da representação cortical do mesmo ${ }^{6}$. Diversas técnicas têm sido utilizadas para a recuperação da função motora nesses pacientes, entretanto existem controvérsias quanto à efetividade das mesmas e há necessidade de novas pesquisas baseadas nos princípios da neuroplasticidade.

Terapia por Restrição e Indução do Movimento

A Terapia por Restrição e Indução do Movimento (TRIM) consiste em uma técnica pela qual o paciente deve reduzir o uso do membro superior contralateral, encorajando a realização de atividades funcionais com o membro superior afetado, a fim de aumentar ou restabelecer a função motora do membro superior deficiente ${ }^{7}$. A primeira parte do protocolo da TRIM consiste da restrição do membro superior não acometida para que as atividades de vida diária do paciente sejam realizadas com o membro superior, a restrição é realizada com um dispositivo em forma de luva por aproximadamente cerca de $90 \%$ do tempo que o paciente permanece em vígilia durante o período de tratamento, dessa forma o paciente durante o tempo de tratamento fica impedido de compensar o uso do membro superior ${ }^{8}$. A segunda parte do protocolo da TRIM consiste do treinamento diário do membro superior por cerca de 6 horas diárias, esse treinamento é supervisionado pelo profissional de reabilitação. $O$ protocolo completo de tratamento é administrado durante o período de 14 dias consecutivos, sendo o treinamento somente administrado durante os 10 dias úteis de tratamento 9 .

\section{Observação de Ação}

A técnica de Observação de Ação consiste em um método onde o paciente assiste a vídeos onde são visualizadas as ações as quais eles estão impossibilitados de realizar corretamente, executando-as logo após ${ }^{10}$. Tal técnica envolve a execução, compreenssão e intenção das ações de si mesmo ou de outras pessoas. Desta maneira são ativados no cérebro os chamados "neurônios espeIho". Estes circuitos neuronais são ativados em determinadas áreas do cérebro, por exemplo, quando estendemos o braço para alcançar um objeto, quando o largamos sobre uma mesa ou quando observamos uma pessoa executando essa mesma ação. Nesse último caso nosso cérebro simula mentalmente a ação visualizada e interpreta a intenção de quem a realizou'11.

\section{Prática Mental}

A Prática Mental consiste em um método de treinamento pelo qual a reprodução interna de um dado ato motor (simulação mental) é repetida extensivamente com a intenção de promover aprendizagem ou aperfeiçoamento de uma habilidade motora. Esta simulação mental (imagética motora) corresponde a um estado dinâmico durante a representação de uma ação específica reativada internamente na memória de trabalho na ausência de qualquer movimento ${ }^{12}$. A Prática Mental representa o resultado do acesso consciente à intenção de um movimento, a qual é geralmente executada de forma inconsciente durante a preparação motora ${ }^{12,13}$, estabelecendo uma relação entre eventos motores e percepções cognitivas ${ }^{14}$. Quando indivíduos são requeridos a realizar a prática mental po- 
dem utilizar duas estratégias diferentes, através de imagens internas ou externas. A imagem interna tem caráter cinestésico com a perspectiva na primeira pessoa, onde a pessoa realiza uma simulação mental tentando sentir o movimento sem que este ocorra. Este tipo de imagem envolve a representação cinestésica para a ação interna. Já a imagem externa é predominantemente visual com a perspectiva tanto para a primeira como para a terceira pessoa, onde a pessoa visualiza o movimento sendo realizado ou por outra pessoa ou por segmentos do seu próprio corpo. Tal tipo de imagem envolve a representação visuo-espacial da ação ou representação visual de um membro em movimento ${ }^{15,16}$.

\section{Ressonância Magnética Funcional e Neuroplasticidade}

Mecanismos neurofisiológicos envolvendo a plasticidade cerebral, como a sinaptogênese (formação sináptica), a arborização dendrítica (brotamento de novos terminais axônicos), o recrutamento de vias córtico-piramidais semelhantes na função, mas anatomicamente distintas das áreas lesionadas e o "desmascaramento" de conexões sinápticas perilesionais funcionalmente silenciosas emergiram da introdução de técnicas não-invasivas em humanos, como, por exemplo, a Ressonância Magnética Funcional (RMF), entre outras ${ }^{17}$. A RMF oferece detalhes anatômicos das estruturas cerebrais em três dimensões, que permitem a reconstrução dos dados através de uma série de cortes tomográficos. $A$ RMF é um método que proporciona uma janela das imagens da atividade neural em múltiplas áreas do SNC, com boa acessibilidade, segurança e alta resolução espacial ${ }^{18}$. Com o uso deste método, é possível observar a existência de alterações significativas na topografia dos mapas somato-motores tanto em sujeitos saudáveis quanto em pacientes. Já que a técnica permite precisar a lesão e seu processo de reorganização plástica segundo as funções alteradas ${ }^{17,19}$, proporcionando melhor compreensão dos mecanismos responsáveis pelos déficits neurológicos e incapacidades irreversíveis ${ }^{20,21}$. Neste contexto, os estudos neurofisiológicos e de neuroimagem fornecem evidências convincentes de que o córtex cerebral humano adulto é capaz de significativa plasticidade funcional após danos ao SNC de diferentes etiologias ${ }^{21}$.

Sendo assim, o presente estudo teve como objetivo verificar a dinâmica da reorganização do sistema nervoso central após a aplicação de técnicas de fisioterapia neurológica, particularmente Terapia de Restrição e Indução do Movimento, Observação Motora e Prática Mental destinadas à recuperação funcional de membros superiores de sujeitos hemiparéticos pós-AVE por meio de estudos que utilizaram imagens de ressonância magnética funcional.

\section{MÉTODO}

Foi realizada revisão nas bases de dados Pubmed/Medline, Cochrane Database of Systematic Reviews, Lilacs, Bireme, Scirus e SciELO. A busca de artigos foi realizada em três idiomas: inglês, espanhol e português. As palavras-chave usadas nas buscas foram: neuroplasticidade, acidente vascular encefálico, membro superior, fisioterapia, reabilitação e imagem por ressonância magnética funcional. Foram utilizadas também essas palavras nos outros idiomas. O período estabelecido para a busca de estudos foi janeiro de 2002 a fevereiro de 2008. Os critérios de inclusão foram: estudos do tipo ensaios clínicos e revisões sistemáticas relacionados à neuroplasticidade e técnicas de fisioterapia neurológica, particularmente Terapia de Restrição e Indução do Movimento, Observação de Ação e Prática Mental, que, por meio de imagens de ressonância magnética funcional, verificaram recuperação funcional de membros superiores de sujeitos hemiparéticos pós-AVE.

\section{RESULTADOS}

Foram encontrados poucos estudos relacionados à neuroplasticidade e técnicas de fisioterapia neurológica que, por meio de imagens de ressonância magnética funcional, verificaram recuperação funcional de membros superiores de sujeitos hemiparéticos pós-AVE. O primeiro teve como objetivo investigar, por meio da TRIM, quatro hemiparéticos crônicos pós-AVE contra um grupo controle de cinco sujeitos sadios. A RMF préintervenção mostrou um padrão de atividade menos significativo no córtex motor contralateral nos pacientes em comparação aos sujeitos sadios devido a uma tendência de aumento na atividade do córtex motor ipsilateral. $\mathrm{O}$ teste de função motora (Motor Activity Log) demonstrou que os pacientes alcançaram ganhos significativos na funcionalidade do membro superior afetado (detectado pelo Motor Activity Log) e redução significativa de comprometimento motor (detectado pela Fugl-Meyer 
Stroke Scale Wolf e pelo Motor Function Test), imediatamente após a TRIM, e esses efeitos persistiram ainda por mais seis meses de acompanhamento. Os efeitos da TRIM foram associados com uma tendência a uma redução na lateralidade do momento pré-intervenção ao momento imediatamente pós-intervenção e seis meses pós-intervenção. $\mathrm{O}$ movimento da mão afetada não foi acompanhado por movimento espelho durante RMF e medidas eletromiográficas de recrutamento espelho sob condições simuladas na RMF não obtiveram valores significativos de correlação com os valores do índice de lateralidade. Estes dados evidenciam que os ganhos funcionais gerados pelo treinamento da TRIM em pacientes crônicos pós-AVE podem estar associados a uma mudança na lateralidade na ativação do córtex motor em direção ao hemisfério não danificado 22 .

Em pesquisa realizada, a RMF foi utilizada para investigar a reorganização cortical em pacientes crônicos de AVE antes e após a TRIM ${ }^{23}$. Participaram seis pacientes (uma mulher e cinco homens com média de idade de 70,3 anos) com AVE isquêmico no hemisfério esquerdo, acometidos há 1,5 ano antes do estudo, e que não apresentaram meIhoras motoras nos últimos três meses antes de iniciar a TRIM. A técnica de TRIM objetiva estimular o paciente a usar a mão afetada. A mão não afetada é posicionada numa órtese por $90 \%$ do tempo no qual o paciente permanece acordado. $O$ tratamento foi focado na realização de tarefas diárias que necessitam do uso da mão. Os pacientes recebiam a TRIM por no mínimo seis horas por dia, supervisionados por um fisioterapeuta. A função motora da mão aumentou em todos os indivíduos após a TRIM. As comparações pré e pós-treinamento revelaram mudanças na ativação (aumento e diminuição) no córtex sensório-motor primário, que dividiram os pacientes em dois grupos: no grupo 1 [três pacientes que apresentavam a área da mão na área motora primária (M1) e suas fibras motoras descendentes intactas, e potencial motor evocado normal na mão afetada] a ativação diminui significativamente no córtex sensório-motor primário lesionado, com aumento da excitabilidade intracortical. Esse padrão reflete um aumento na eficiência sináptica que não era possível nos pacientes com projeções danificadas da M1. No outro grupo (três pacientes com $\mathrm{M} 1$ e região piramidal descendente lesada) a ativação no córtex sensório-motor primário lesionado aumentou significativamente após a
TRIM, acompanhada pela excitabilidade intracortical diminuída. Isso sugere que o aumento na eficiência sináptica não é possível, o que conduz para reorganização na extensão, transferência e recrutamento adicional do trabalho de áreas corticais sensório-motoras.

Emoutra investigação, pesquisadores realizaram um estudo utilizando a técnica da contrição e indução modificada (mTRIM) objetivando verificar a reorganização cortical após utilização da técnica. Participaram quatro pacientes, sendodois homense duas mulheres com idade média de 60,5 anos; com AVE unilateral ocorrido num período anterior a um ano do estudo, com déficit motor moderado. A técnica modificada consistia de 30 minutos de terapia enfatizando o braço afetado em atividades diárias, durante três dias por semana por um período de 10 semanas. O braço não afetado era restringido cinco dias da semana por cinco horas. Antes da aplicação os sujeitos nunca ou ocasionalmente utilizavam o membro afetado nas atividades diárias. Após 10 semanas de tratamento os pacientes apresentaram modificações, comparadas ao pré-teste na RMF. Um paciente não obteve ganhos funcionais com a técnica e também não demonstrou mudanças no sinal da RMF. Os outros três apresentaram alteração da atividade cerebral após o tratamento, com ativação adicional no hemisfério contralateral (giros pré e pós-central, e frontal inferior ao médio). Além disso, os sujeitos que apresentaram alterações no sinal da RMF mostraram ganhos funcionais das atividades avaliadas com a mão afetada ${ }^{6}$.

Em outro estudo clínico, quatro pacientes com AVE (uma mulher e o restante do sexo masculino, média de idade 62,75 anos), com hemiparesia moderada do membro superior, foram divididos em três grupos, de forma aleatória, para tentar verificar a praticabilidade de usar a técnica de Prática Mental associada com a TRIM, para obtenção de ganhos funcionais nestemembroapós duas semanas de tratamento. A RMF foi utilizada para estabelecer mudanças na oxigenação cerebral frente às diferentes intervenções adotadas. Um grupo treinou apenas a Prática Mental (após o treino houve ativação cerebelar direita notável que não foi apresentada anteriormente), o outro a técnica de TRIM (apresentou, pós-treinamento, ativação cortical bilateral aumentada nas áreas motora e pré-motora) e no último foram associadas às duas técnicas (ativação contralateral mais focal no córtex motor primário). Os autores perceberam que a prática física e mental em 
conjunto conduza uma melhorafuncional ealteram a ativação cortical, mas não conseguiram concluir se esse tipo de terapia combinada rende mais desempenho motor que a TRIM sozinha ${ }^{24}$.

Estudos laboratoriais analisaram oito pacientes com AVE crônico (mais de seis meses) e média de idade de 57 anos. Estes pacientes apresentavam déficits moderados do membro superior em conseqüência de infarto da artéria cerebral média. Realizaram a terapia de "observação de ação" por 18 sessões, a qual consistia em assistir um vídeo contendo ações do braço nas atividades de vida diárias, seguidas de repetições das ações observadas. Verificou-se uma melhora motora em um tratamento de quatro semanas. O grupo controle assistiu a uma fita de vídeo contendo figuras geométricas e em seguida realizaram as mesmas atividades que o grupo experimental. O grupo controle não demonstrou nível de significância nas trocas de ativação entre pré e pós-tratamento. Em contraste, o grupo experimental demonstrou numerosas diferenças. No pós-tratamento, aumentos na ativação foram observados em áreas sensório-motoras incluindo a área motora suplementar, o córtex motor ventral bilateral, as áreas parietal superior e inferior bilateralmente e o cerebelo. Também ocorreram aumentos da ativação após o treino quando manipulações complexas foram executadas com a mão não afetada. Ativação contralateral à lesão incluiu o lóbulo parietal superior e inferior e o cerebelo, ativações no lado lesionado no córtex ventral pré-motor e cerebelo. A mais importante mensuração dos efeitos da terapia foi à comparação das trocas de ativação entre os grupos. Ativação significante foi identificada no hemisfério não afetado no córtex pré-motor ventral, área motora suplementar, insula e giro temporal superior, e no hemisfério afetado córtex prémotor ventral, giro supra-marginal e giro temporal superior. Além das trocas na ativação cerebral, o grupo experimental também demonstrou melhoras funcionais significantes quando comparadas ao controle. Os autores concluíram que a observação da ação tem um impacto adicional positivo na recuperação das funções motoras pós-AVE pela reativação das áreas motoras, que contêm o sistema combinado de execução e observação da ação. Suportam também a noção de que a ativação de representações centrais referentes às ações pode constituir um mecanismo poderoso para a recuperação de déficits motores pós-AVE. Sabe-se que a observação da ação recruta áreas no sistema nervoso espelho, como uma experiência da função motora e competência das ações observadas ${ }^{25}$.

\section{CONSIDERAÇÕES FINAIS}

De acordo com a pesquisa realizada, foi possível verificar ativações ipsilateral ou contralateral à lesão, ativações bilaterais, bem como ativações em torno da área lesionada. Em um estudo de revisão abordando os mecanismos de reorganização cerebral pós-AVE através da RMF ${ }^{26}$, demonstrando que durante o movimento da mão afetada ocorre geralmente um aumento na ativação dentro do córtex motor primário no lado lesado, córtex dorsal prémotor, e da área motora suplementar, em paralelo à melhora na funcionalidade ${ }^{18}$. Estudos apontam para a relevância do papel do córtex motor primário na recuperação funcional. O mesmo lado do córtex motor primário intacto com projeções ao córtex ipsilateral contribuem para tal recuperação. Uma explanação é que a interrupção das projeções de M1 para o cordão espinal motor conduz ao recrutamento aumentado de áreas secundárias motoras com suas próprias projeções para o cordão espinhal motor dos neurônios. Essas projeções secundárias ao cordão espinal são menos numerosas e menos excitatórias que para M1 e, portanto, apresentam recuperação inferior nos pacientes que dependem dessas regiões para gerar a saída motora ${ }^{26}$. Em recente estudo clínico foram observadas evidências de que a ativação no hemisfério contralateral é alta durante o movimento da mão afetada no estágio subagudo após o AVE, declina com o passar do tempo e restam algumas extensões por anos após o AVE ${ }^{27}$. Os resultados são limitados se realmente as regiões secundárias são relevantes na recuperação, mas o recrutamento e a adaptação de áreas motoras secundárias sobreviventes em ambos os hemisférios podem ajudar pacientes a conseguirem melhores resultados ${ }^{26}$. Trabalho recente relata que é complexo traçar-se estratégias eficazes após um AVE devido aos diversos fatores que influenciam na manifestação da lesão e na própria recuperação ${ }^{28}$. A associação de técnicas de alta resolução espacial, como a RMF, com exames de alta resolução temporal, como os potenciais evocados somatossensoriais, têm demonstrado que a limitações destas técnicas podem ser sanadas justamente por estas interações. Estudo atual mostra que, com esta associação de técnicas, mesmo em modelos animais, pode-se observar durante a recuperação de lesão 
induzida um acoplamento de áreas corticais e estado neurovascular indicativo de recuperação. Estes achados podem facilitar os entendimentos futuros sobre atividades simultâneas no córtex (possível acoplamento de fases elétricas) e sobre os processos de neovascularização encefálica ${ }^{29}$.

Com a modernidade dos equipamentos de imagem, como a RMF, e a observação dos estudos pesquisados quanto às mudanças na ativação cerebral, os resultados mostraram-se inconsistentes, talvez por diferentes características entre os pacientes (local e extensão da lesão, estágio pós-AVE, terapia realizada e graus de recuperação). Possivelmente o cérebro encontra diferentes vias para uma reorganização, dependendo da área lesionada e das abordagens terapêuticas utilizadas. Embora se tenham verificado mudanças funcionais no membro superior e plasticidade cerebral em diferentes pacientes e abordagens, ainda não foi encontrada a melhor técnica e forma de reabilitação para o membro superior.

\section{REFERÊNCIAS}

1.Cicerone KD, Dahlberg C, Malec JF, Langenbahn DM, Felicetti T, Kneipp S, et al. Evidence-based cognitive rehabilitation: updated review of the literature from 1998 through 2002. Arch Phys Med Rehabil 2005;86:1681-92.

2.Stewart DG. Stroke rehabilitation. Epidemiologic aspects and acute management. Arch Phys Med Rehabil 1999;80:S4-7. 3.Alexandrova ML, Bochev PG. Oxidative stress during the chronic phase after stroke. Free Radic Biol Med 2005;39:297316.

4.Haase VG, Lacerda SS. Neuroplasticidade, variação interindividual e recuperação funcional em neuropsicologia. Tem Psicol SBP 2003;11:28-42.

5.Cauraugh JH, Summers JJ. Neural plasticity and bilateral movements: A rehabilitation approach for chronic stroke. Prog Neurobiol 2005;75:309-20.

6.Szaflarski JP, Page SJ, Kissela BM, Lee J, Levine P, Strakowski SM. Cortical Reorganization Following Modified Constraint-Induced Movement Therepy: A Study of 4 Patients With Chronic Stroke. Arch Phys Med Rehabil 2006;87:1052-8.

7.Mark VW, Taub E, Morris DM. Neuroplasticity and constraintinduced movement therapy. Eura Medicophys 2006;42:26984.

8.Taub E, Uswatte G, Mark VW, Morris DM. The learned nonuse phenomenon: implications for rehabilitation. Eura Medicophys 2006;42:241-56.

9.Morris DM, Taub E, Mark VW. Constraint-induced movement therapy: characterizing the intervention protocol. Eura Medicophys 2006;42:257-68.

10.Fabbri-Destro M, Rizzolatti G. Mirror neurons and mirror systems in monkeys and humans. Physiology (Bethesda) 2008;23:171-9.

11.Rizzolatti G, Sinigaglia C. Mirror neurons and motor intentionality. Funct Neurol 2007;22:205-10.
Dessa maneira, os resultados não podem ser generalizados devido às diferentes características dos pacientes e ao pequeno número de indivíduos em cada estudo. Sugere-se, então, que sejam realizados mais estudos associando técnicas de fisioterapia neurológica a imagens de ressonância magnética funcional, pois os resultados indicam que a experiência comportamental após lesão é o principal modulador das mudanças neurofisiológicas e neuroanatômicas. Sendo assim, nosso estudo tem como limitação o pequeno número de pesquisas associando imagens de ressonância e possíveis alterações plásticas corticais induzidas pelas técnicas da fisioterapia neurológica. Mesmo assim, parece evidente que estas técnicas geram alterações plásticas de forma associada às melhoras nos padrões motores $^{30,31}$.

12.Decety J, Grèzes J. Neural mechanisms subserving the perception of human actions. Trends Cogn Sci 1999;3:172-8.

13.Lotze M, Cohen LG. Volition and imagery in neurorehabilitation. Cogn Behav Neurol 2006;19:135-40.

14.Jackson PL, Doyon J, Richards CL, Malouin F. Potential role of mental practice using motor imagery in neurological rehabilitation. Arch Phys Med Rehabil 2001;82:1133-41.

15.Deiber MP, Ibanez V, Honda M, Sadato N, Ramans R, Hallett $M$. Cerebral processes related to visuomotor imagery and generation of finger movements studied with positron emission tomography. Neurolmage 1998;7:73-85.

16.Ruby P, Decety J. Effect of subjective perspective taking during simulation of action: a PET investigation of agency. Nat Neurosci 2001;4:546-50.

17.Gómez-Fernández L. Plasticidad cortical y restauración de funciones neurológicas: una actualización sobre el tema. RevNeurol 2000;31:749-56.

18.Rocca MA, Filippi M. Functional MRI to study brain plasticity in clinical neurology. Neurol Sci 2006;27:24-6.

19.Díaz-Arribas MJ, Pardo-Hérvas $P$, Tabares-Lavado M, RiosLago M, Maestú F. Plasticidad del sistema nervioso central y estratégias de tratamiento para la reprogramación sensoriomotora: comparaciónde dos casos de accidente cerebrovascular isquêmico en el territorio de la arteria cerebral media. Rev Neurol 2006;42:153-8.

20. Hodics T, Cohen LG, Cramer SC. Functional Imaging of Intervention Effects in Stroke Motor Rehabilitation. Arch Phys Med Rehabil 2006;87:S36-42.

21. Hidler J, Hodics T, Xu B, Dobkin B, Cohen LG. MR compatible force sensing system for real-time monitoring of wrist moments during fMRI testing. J Neurosci Met 2006;155:300-7. 
22.Schaechter JD, Kraft E, Hilliard TS, Dijkhuizen RM, Benner $\mathrm{T}$, Finklestein SP, et al. Motor recovery and cortical reorganization after constraint-induced movement therapy in stroke patients: a preliminary study. Neurorehabil Neural Repair 2002;16:326-38.

23.Hamzei F, Liepert J, Dettmers C, Weiller C, Rijntjes M. Two different reorganization patterns after rehabilitative therapy: an exploratory study with FMRI and TMS. Neuroimage 2006;31:710-20.

24.Butler AJ, Page SJ. Mental Practice with Motor Imagery: Evidence for Motor Recovery and Cortical Reorganization after Stroke. Arch Phys Med Rehabil 2006;87:S2-S11.

ce imaging, electrophysiology, and behavioral testing in rats. J Neurosci 2008;28:1022-9.

25.Ertelt D, Small S, Solodkin A, Dettmers C, McNamara A, Binkofski $F$, et al. Action observation has a positive impact on rehabilitation of motor deficits after stroke. Neurolmage 2007;36:164-73.
26.Ward NS. Functional reorganization of the cerebral motor system after stroke. Curr Opin Neurol 2004;17:725-30.

27.Gerloff C, Bushara K, Sailer A, Wassermann E, Chen R, Waldvogel $D$, et al. Multimodal imaging of brain reorganization in motor areas of the contralesional hemisphere of well recovered patients after capsular stroke. Brain 2006;129:791-808.

28.Cheatwood JL, Emerick AJ, Kartje GL. Neuronal plasticity and functional recovery after ischemic stroke. Top Stroke Rehabil 2008;15:42-50.

29.Weber R, Ramos-Cabrer P, Justicia C, Wiedermann D, Strecker $C$, Sprenger $C$, et al. Early prediction of functional recovery after experimental stroke: functional magnetic resonan 30.Stinear CM, Barber PA, Coxon JP, Fleming MK, Byblow WD. Priming the motor system enhances the effects of upper limb therapy in chronic stroke. Brain 2008;131:1381-90.

31.Gauthier LV, Taub E, Perkins C, Ortmann M, Mark VW, Uswatte G. Remodeling the brain: plastic structural brain changes produced by different motor therapies after stroke. Stroke 2008;39:1520-5. 